\title{
Ultrasound-guided block of sciatic and femoral nerves: an anatomical
}

\section{study}

Sonja Waag ${ }^{1}$, Michael H. Stoffel ${ }^{2}$, Claudia Spadavecchia ${ }^{3}$, Urs Eichenberger ${ }^{4}$, Helene Rohrbach $^{3,5}$

${ }^{1}$ Orthopaedic Center for Musculoskeletal Research, Department of Orthopaedic Surgery, Koenig-Ludwig-Haus, Julius-Maximilians-University, Wuerzburg, Germany

${ }^{2}$ Division of Veterinary Anatomy, Vetsuisse Faculty University of Bern, Switzerland

${ }^{3}$ Vetsuisse Faculty, Department of Clinical Veterinary Medicine, Anaesthesia Department, Bern,

Switzerland

${ }^{4}$ Department of Anaesthesia, Intensive Care and Pain Medicine, St. Anna Clinic, Lucerne,

Switzerland

${ }^{5}$ Graduate School for Cellular and Biomedical Sciences, University of Bern, Switzerland

Correspondence: H. Rohrbach, Anaesthesiology Section, Department of Veterinary Sciences, Vetsuisse Faculty, University of Berne, Laenggassstrasse 124, 3012 Bern, Switzerland.

E-mail: helene.rohrbach@vetsuisse.unibe.ch

Fax: +413163122 26,

Phone: +41316312315 


\section{Abstract}

2 The sheep is a popular animal model for human biomechanical research involving invasive 3 surgeries of the hip or the stifle joint. These painful procedures can only be ethically justified 4 with the application of adequate analgesia protocols. Regional anaesthesia as an adjunct to 5 general anaesthesia may markedly improve wellbeing of these experimental animals during the 6 post-operative period due to a higher analgesic efficacy when compared to systemic drugs and $7 \quad$ therefore reduce stress and consequently the severity degree of such studies.

8 As a first step fourteen sheep cadavers were used to establish a new technique of peripheral 9 blockade of the sciatic and the femoral nerves under sonographic guidance and to evaluate the 10 success rate by determination of the colorization of both nerves after injection of $0.5 \mathrm{ml}$ of $0.1 \%$ 11 methylene blue solution. First, both nerves were visualized sonographically. Then, methylene 12 blue solution was injected and subsequently, the length of colorization was measured by gross 13 anatomical dissection of the target nerves. Twenty-four sciatic nerves were identified 14 sonographically in 12 out of 13 cadavers. In one animal, the nerve could not unequivocally be 15 ascertained and, consequently, nerve colorization failed. Twenty femoral nerves were located 16 by ultrasound in 10 out of 13 cadavers. In 3 cadavers, signs of autolysis impeded the scan. This 17 study provides a detailed anatomical description of the localization of the sciatic and the femoral 18 nerves and presents an effective and safe yet simple and rapid technique to perform peripheral 19 nerve blocks with a high success rate. 


\section{Introduction}

The sheep is a valuable animal model for biomechanical and translational research due to a rather inexpensive husbandry and easy handling ${ }^{1-4}$. Analogies between various ovine and human joints as well as a high comparability of the ovine bone morphology to the human skeleton enable not only studies concerning the replacement of ligaments but also the treatment of chondral and osteochondral defects ${ }^{5,6}$. Many of the surgical procedures are highly invasive and painful thus requiring adequate peri-operative pain management to ensure post-operative well-being $^{7-11}$.

The use of loco-regional anaesthesia techniques allows the depth of anesthesia to be reduced during surgery and decreases the need for systemic analgesic drugs during and after surgery due to their long-lasting analgesic efficacy ${ }^{7,12}$. In humans, application of such techniques often allows the induction of general anaesthesia to be circumvented. Furthermore, the risk of adverse effects such as vomiting and nausea is minimized during the postoperative phase while pain is reduced and patients can be released from the hospital earlier ${ }^{13-15}$. In most animals, the need of unconsciousness during orthopedic surgery remains unavoidable due to a lack of compliance.

A large variety of regional anaesthesia techniques has been described in dogs and cats ${ }^{16-18}$. In small ruminants, only a few methods have been evaluated so far, spinal administration of analgesic drugs being the technique of choice for surgery on the hind limb ${ }^{19-21}$. However, bilateral motor blockade can cause severe stress during recovery ${ }^{20,22,23}$. The intra-articular administration of analgesic drugs leads to effective analgesia during and after surgery involving stifle joints ${ }^{24,25}$. Though, cytotoxic effects of local anaesthetics on articular chondrocytes have recently been demonstrated in vitro ${ }^{26}$. 
The sciatic and the femoral nerves are the main nerves of the hind limb. A peripheral blockade of these nerves allows surgery of the knee and the tibia to be performed and, therefore, is a popular technique in human and small animal medicine ${ }^{27,28}$. The innervation of the hip joint is species specific. In the dog, this joint is innervated by the sciatic and the femoral nerves, the latter apparently containing both motor and sensory fibers, and the glutaeus cranialis nerve with a pure motor function ${ }^{29}$. In humans and horses, the obturator nerve, which is also considered to be purely motor, is involved as well ${ }^{30,31}$. As for the ruminants, no species specific information has been published so far. Until now, the neuraxial administration of analgesic drugs was the only technique allowing effective anaesthesia of the hip joint in sheep ${ }^{21}$ as a very proximal blockade of the sciatic nerve is needed to block sensory afferents from this area. In humans and dogs, the paravertebral or parasacral administration of local anaesthetics to the sciatic nerve leads to an adequate level of peri-operative analgesia of the hip joint ${ }^{32,33}$. This approach reduces the potential for cardiovascular side effects such as hypotension and bradycardia and reduces the risk for postoperative complications as compared to epidural anaesthesia ${ }^{34}$. As a consequence, postoperative well-being may be improved in animals and humans ${ }^{7,35,36}$. Several anatomical landmarks can be used for the perineural administration of local anaesthetics to the sciatic and the femoral nerves. Notwithstanding, the success rate of these blocks could be further increased even with smaller volumes of injected local anaesthetics when using a nerve stimulator ${ }^{17,36}$. Lately, the technique has been further developed and perineural injections can now be performed under sonographic guidance. Various ultrasound guided locoregional anaesthesia techniques have been established in human and small animal medicine ${ }^{37-}$ under ultrasound guidance has not yet been described in sheep. 
68 The direct visualization of the needle, of the target nerve as well as of the surrounding 69 anatomical structures such as blood vessels, bone, muscles, tendons and fat tissue allows exact positioning of the needle tip close to the targeted nerve. Due to real-time visualization of 71 the spreading of the local anesthetic, the drug volume can be minimized. A precise localization of the target nerve leads to a faster onset of blockade, longer duration of effect and therefore

73 better quality of nerve blockade ${ }^{42}$.

74 As a first step in the process of establishing a new technique, it is useful to inject dye into 75 cadavers in order to assess the success rate by staining the target nerves $37,38,43$.

76 The aim of this study was to establish the technique of perineural injection of methylene blue 77 close to the sciatic and femoral nerves under ultrasound-guidance in sheep cadavers and to evaluate the distribution of a minimal amount of methylene blue solution at the desired

79 locations. We hypothesized that it would be possible to visualize the sciatic and the femoral 80 nerves by ultrasound in adult sheep cadavers and that the injection of a small volume of 81 methylene blue would lead to a distinct colorization of the target nerves. 


\section{Materials and methods}

\section{Animals}

85 Fourteen cadavers of adult female Swiss Alpine Sheep with a mean weight of $72(56-83) \mathrm{kg}$ 86 were used in this study. The animals were euthanized at the end of an experimental 87 cardiovascular trial.

88 Both hind limbs, the pelvis and the lumbar spine were abscised in toto before freezing for 2-8 89 weeks. The cadavers were thawed for 36 hours at room temperature before starting the 90 experiment.

\section{$91 \quad$ Pilot study}

92 In one sheep, the anatomy of the sciatic and the femoral nerves was assessed and compared 93 with the structures previously identified by ultrasound (linear array probe, 6-13 Mhz; MTurbo®, 94 Sonosite, Seattle, USA).

\section{Procedures}

After "sternal" positioning of the cadavers, the skin and subcutaneous fat tissue were gently removed as it had become hard and dry due to the freezing process. The sacrum and the tuber coxae were palpated and used as anatomical landmarks. A linear array probe (6-13 Mhz; MTurbo $\AA$, Sonosite, Seattle, USA) was positioned on the tuber coxae at an angle of approximately $70^{\circ}$ to the long axis of the cadaver and moved in caudal direction until the sciatic nerve could be visualized where it leaves the foramen ischiadicum majus. A needle specifically designed for injections under ultrasound guidance $(22 \mathrm{G} \times 80 \mathrm{~mm}$; SonoTAP cannula $($, Pajunk, Germany) was positioned in-plane in latero-medial direction until the needle tip could be detected in close proximity to the nerve (Fig.1). Methylene blue solution $(0.5 \mathrm{ml} ; 0,1 \%)$ was then injected under sonographic control. Due to the small volume, the space being occupied by the injected fluid was not visible. 
107 In a second step, the cadaver was positioned in supine position. The sartorius muscle at the 108 midpoint of the medial aspect of the thigh was used as an anatomical landmark. The probe was 109 positioned perpendicular to the long axis of the thigh upon the sartorius muscle and was moved 110 in a proximal and caudal direction until the femoral artery, the vein and the nerve could be 111 visualized under the fascia of the sartorius muscle as proximally as possible. The needle was 112 positioned in-plane until the tip of the needle reached the targeted structures (Fig.2). Directly 113 after injection of the methylene blue, the quality of colorization of the target nerves was 114 quantified by measurements of the length of colorization after dissection of both nerves. A photo 115 of the colored tissue with a scale was taken to allow later evaluation (Fig.3, 4). 


\section{Results}

\section{Pilot study}

119 The sciatic nerve was identified sonographically in the foramen ischiadicum majus of the pilot 120 animal (Fig.1). Therefore, the tuber coxae and the tuber ischiadicum served as the most important anatomical landmarks. When a line was drawn from the tuber coxae to the tuber ischiadicum, the injection site could be located in the middle of this line.

123 The sartorius muscle with its triangular appearance and its fascia as the limit between the 124 muscle, nerve and vessels were identified as the most important anatomical landmark for the 125 femoral nerve (Fig.2). The nerve was located underneath the fascia but superficial to the 126 femoral artery and vein.

\section{Main study}

128 We were able to identify 24 sciatic nerves in 12 out of 13 sheep cadavers using ultrasound.

129 They appeared as oval, hypoechoic structures with a hyperechoic rim (Fig.1). The cadavers 130 were dissected after injection of methylene blue: 10 sciatic nerves could be stained perineurally using methylene blue over a mean length of $4 \mathrm{~cm}, 8$ sciatic nerves were stained either perineurally or the nerve itself over a mean length of $3 \mathrm{~cm}$ (Fig.3), and 6 sciatic nerves were stained over a mean length of $4 \mathrm{~cm}$.

134 Twenty femoral nerves were identified in 10 out of 13 sheep cadavers underneath the fascia of the sartorius muscle (Fig.2): in 6 femoral nerves adequate scanning and perineural injections were not possible due to signs of autolysis, in 6 femoral nerves, dye was found perineurally over a mean length of $3 \mathrm{~cm}$ and in 14 femoral nerves, the mean length of staining was $4 \mathrm{~cm}$ (Fig.4). Again, slight neural staining was expected while every effort was made to avoid intraneural injections. 


\section{Discussion}

142 In an effort to further improve analgesia, we evaluated the sciatic and femoral nerve block under 143 ultrasound guidance with encouraging results. With this study we could demonstrate the 144 feasibility to block the sciatic and the femoral nerves in sheep with a high success rate. 145 Ultrasound guidance was very useful in detecting and staining the sciatic and femoral nerve 146 perineurally. The results of this study promise effective blockade of the sciatic and femoral nerves under ultrasound guidance for orthopaedic surgery in sheep.

\section{Anatomy}

149 The sciatic nerve leaves the spine from the segments L6 to S2 of the plexus lumbosacralis and 150 exits the pelvis through the foramen ischiadicum majus. It courses between the gluteus medius and profundus muscles before bending distally between the trochanter major of the femur and the tuber ischiadicum ${ }^{44,45}$. The nerve progresses on the caudal side of the thigh. In the middle of the thigh it splits into the tibial and peroneus communis nerves which run caudally to the fossa poplitea ${ }^{46}$.

After leaving the pelvic cavity, the nerve crosses the gemelli muscles between the trochanter major of the femur cranially and the tuber ischiadicum caudally. Along this way, the sciatic nerve sends motor branches to the gluteus profundus muscle, the gemelli muscles and the quadratus femoris muscle. The sciatic nerve is the most important sensory nerve of the hip joint as it dispatches nerve branches to the hip joint capsule ${ }^{46}$. The nerve passes distally to the plantar side of the thigh, deep to the biceps femoris muscle ${ }^{44}$. In the middle of the thigh, the sciatic nerve splits into the tibial and peroneus communis nerves ${ }^{46}$. The desensitization of the hip joint requires a very proximal block of the sciatic nerve in order to block its branches to the hip joint, too ${ }^{33,47}$. Between the acetabulum and the sacrum, the sciatic 
165

hyperechoic rim. Due to the very deep position of the sciatic nerve and the limited depth of penetration of the ultrasound waves, the sciatic nerve can only be visualized near the foramen ischiadicum majus in sheep by using a linear high frequency transducer.

For surgery in the area of the stifle joint and more distal structures, an additional blockade of the femoral nerve is required ${ }^{7,40}$.

The femoral nerve originates from the segments $L 4$ to $L 6$ of the plexus lumbalis and reaches the lacuna musculorum in conjunction with the iliopsoas muscle. Distally to these structures, it splits off the saphenous nerve as the main sensory nerve for the stifle joint and exits the abdominal cavity through the lacuna musculorum which is delimited by the ligamentum inguinale and the fascia iliaca. It then enters the quadriceps femoris muscle where it gives off branches innervating the quadriceps femoris muscle ${ }^{45,46}$. Close to its origin, the saphenous nerve sends motor branches to the sartorius, pectineus and gracilis muscles and courses in the femoral triangle embedded between the vastus medialis muscle and the sartorius muscle in compound with the femoral artery and vein under the fascia of the sartorius muscle near the caudal margin of the femur between its proximal and middle third ${ }^{7,40,44,45,48}$. In this trial, the femoral nerve could not be differentiated from blood vessels due to the lack of blood flow in the cadavers. Consequently, the triad of femoral artery and vein and the saphenous nerve could not be resolved visually from each other.

The femoral nerve can be blocked at the paravertebral level (lumbosacral block). With this approach, local anesthetic needs to be injected to the roots of the femoral nerve at three or four injection points ${ }^{18,33}$. At the mid-femoral level, the saphenous nerve can be blocked with one single injection ${ }^{7,40,48}$.

The peripheral blockade of the sciatic and saphenous nerve allows effective desensitization of tissue at the hind limbs, especially at the stifle joint and the tibia ${ }^{7,40}$. The decisive factor is the 
localization of the nerve. Anatomical landmarks to block the sciatic and the saphenous nerves

190 under ultrasound guidance or by use of a neuro-stimulator have been evaluated. In most trials,

191 the sciatic nerve was blocked at the "mid-femur" before the peroneus communis nerve and the

192 tibial nerve diverge near the stifle joint ${ }^{38,41}$. The femoral nerve was blocked at the medial aspect

193 of the thigh, distally to the pectineus muscle but cranially to the femoral artery and ventrally to

194 the fascia iliaca ${ }^{41}$.

195 Rasmussen et al ${ }^{49}$ successfully blocked the common peroneal and the tibial nerves at the

196 caudal thigh in the groove between the biceps femoris and semimembranosus/semitendinosus

197 muscle at the midpoint between the patella and the greater trochanter. The saphenous nerve

198 was blocked at the medial aspect of the thigh at the midpoint between the pectineus muscle and

199 the medial epicondyle of the femoro-tibial joint between the sartorius and gracilis muscle.

200 With the use of a nerve stimulator, the sciatic nerve can be blocked between the trochanter

201 major and the tuber ischiadicum ${ }^{7,40,43,50}$. The femoral nerve can be blocked at the medial thigh

202 cranial to the femoral artery and caudal to the fascia of the rectus femoris muscle ${ }^{51}$ and medial

203 to the sartorius muscle ${ }^{40}$.

204 In this trial, we chose a very proximal approach to the sciatic nerve in order to block the nerve

205 before branches for the hip joint are given off: parasacrally in the foramen ischiadicum majus

206 where the nerve crosses the ilium. The saphenous nerve was blocked between the proximal

207 and middle thirds of the femur, underneath the sartorius muscle and superficial to the femoral

208 vessels. The sciatic and the femoral nerves are the main nerves of the hind limb and, thus, have

209 to be blocked for hip or stifle joint surgery to ensure adequate peri-operative analgesia.

210 Surprisingly enough, no additional analgesic effect could be detected after blocking these

211 nerves in an experimental study in sheep undergoing a surgical procedure on the stifle joint ${ }^{52}$. 
212 The systemic administration of a large variety of analgesic drugs besides the block to the

213 animals might explain this result.

214 McNamee et al. ${ }^{53}$ evaluated the additional obturator nerve block to the sciatic femoral nerve

215 block in humans undergoing total knee replacement. This study showed an improvement in

216 post-operative analgesia by adding the obturator nerve block. In domestic species, the following

217 spinal nerves provide skin sensation at the stifle joint: the iliohypogastric, ilioinguinal,

218 genitofemoral and cutaneous femoral nerve ${ }^{54}$.

219 To the author's knowledge, the additional obturator nerve block has not yet been described in

220 sheep. However, the obturator nerve is described as pure motor nerve with the exception of the

221 stifle joint in horses ${ }^{46}$. Thus, an obturator nerve block is unlikely to improve overall hind-limb

222 analgesia.

\section{Learning curve}

224 After a very short period of adjustments, the anatomical landmarks were easily identified. The

225 sciatic and femoral nerve blocks were feasible and rapidly performed. A person lacking previous

226 ultrasound experience quickly learned how to use the ultrasound technique and improve

227 accuracy and speed in performing the nerve blocks under ultrasound guidance. The possibility

228 to control each injection in necropsy further accelerated the learning process.

229 A steep learning curve of the needle placement under ultrasound guidance has previously been

230 shown for breast cyst aspiration ${ }^{55,56}$.

\section{Limitations of the study}

232 In two cadavers, the sciatic and the femoral nerves could not be identified because of the

233 limited quality of the cadaveric tissue. Before scanning the cadaver, the skin and the

234 subcutaneous fat tissue of the frozen cadaver had to be removed as it became firm and dry due

235 to the freezing process, thus generating severe artifacts impeding the sonographic view on the 
underlying structures. When the sciatic nerve was evaluated sonographically in an

237 anaesthetized sheep being involved in another experimental trial, the sciatic nerve could easily

238 be identified.

\section{Use of ultrasound}

240 The necessity of an expensive ultrasound machine might limit the application of such a 241 technique in experimental settings. For this trial, a linear array probe $(6-13 \mathrm{MHz})$ was used to 242 allow the best possible visibility of the sciatic and femoral nerves, but due to the very profound 243 position of the sciatic nerve and the embedding in the muscles, the quality of the ultrasound 244 image was reduced ${ }^{42}$. The use of probes with lower frequency would have led to increased 245 depth of visualization but also to a loss of detail visibility.

246 The in-plane technique used in the present study offers significant advantages over the out-of247 plane technique as the tip of the needle and the shaft can be visualized while advancing in 248 direct proximity to the target nerve.

249 The femoral nerve is lying relatively superficial. Anyway, the identification was difficult due to the 250 lack of blood flow through the femoral vessels and the impossibility to differentiate the neural 251 structures from the vessels.

252 The success rate of peripheral nerve blocks can be significantly increased with the use of 253 ultrasound guidance as compared to the application of a nerve stimulator ${ }^{40,42,57}$. Intraneural 254 injections of local anaesthetics can lead to nerve damage and should be avoided ${ }^{58}$. Therefore, 255 complete neural staining was not expected with the minute volumes used.

256 The use of needles designed for injections under ultrasound guidance with reflector marks allow 257 an even more precise localization of the needle tip. Consequently, with the combination of 258 modern sonographic accessories and the in-plane technique, a successful perineural injection 259 of analgesic drugs can be provided. 
260 As intended in this trial, the small volumes of methylene blue usually stained the perineural 261 tissue instead of the nerve itself. Therefore, intraneural injection of methylene blue could be 262 excluded.

263 The experiment described here was a pure cadaveric study. Therefore, the analgesic efficacy of 264 the sciatic and femoral nerve block could not be evaluated. However, the proof of principle of 265 both nerve blocks in dogs, humans and goats to desensitize the stifle joint has been provided 266 before ${ }^{7,40,59}$. To the author's knowledge, the effectiveness of a single parasacral sciatic block 267 for hip surgery has not been demonstrated so far, neither in humans nor in animals.

268 As a next step it will be necessary to test the described regional anaesthesia techniques in 269 animals undergoing orthopaedic surgery. Even though the duration of effects of local 270 anaesthetics is longer in sheep than in humans, a perineural catheter will be helpful to prolong 271 the post-operative analgesia for several days.

\section{Conclusions}

274 With this study we provide an exhaustive anatomical description of the localization of the sciatic and femoral nerve by using ultrasound imaging in sheep. The evaluated technique is simple and rapid to perform and produces precise and clear images of the nerves. In vivo studies will be necessary to assess the efficacy of this technique in providing satisfactory perioperative sensory analgesia and motor blockade.

\section{Conflict of interest statement}

281 None of the authors of this paper has a financial or personal relationship with other people or organisations that could inappropriately influence or bias the content of the paper. 


\section{Funding}

285 The project was partly funded by the 3R foundation.

286 


\section{References}

1. Kelly BT, Robertson W, Potter HG, et al. Hydrogel meniscal replacement in the sheep knee: preliminary evaluation of chondroprotective effects. The American journal of sports medicine. 2007 Jan;35(1):43-52.

2. Raske TG, Pelkey S, Wagner AE TA. Effect of intravenous ketamine and lidocaine on isoflurane requirement in sheep undergoing orthopedic surgery. Lab Animal. 2010;39(3):76-79.

3. Turner a S. Experiences with sheep as an animal model for shoulder surgery: strengths and shortcomings. Journal of shoulder and elbow surgery / American Shoulder and Elbow Surgeons . [et al]. 2007;16(5 Suppl):S158-63.

4. Martini L, Fini M, Giavaresi G, et al. Overview Sheep Model in Orthopedic Research : A Literature Review. Comparative medicine. 2001;51(4):292-299.

5. Allen MJ, Houlton JE, Adams SB, Rushton N. The surgical anatomy of the stifle joint in sheep. Vet Surg [Internet]. 1998;27(6):596-605. Available from: http://www.ncbi.nlm.nih.gov/entrez/query.fcgi?cmd=Retrieve\&db=PubMed\&dopt=Citation \&list_uids=9845224

6. Phillips TW, Gurr KR, Rao DR. Hip implant evaluation in an arthritic animal model. Archives of orthopaedic and trauma surgery [Internet]. 1990 Jan [cited 2013 Aug 16];109(4):194-6. Available from: http://www.ncbi.nlm.nih.gov/pubmed/2383447

7. Adami C, Bergadano A, Bruckmaier R, et al. Sciatic-femoral nerve block with bupivacaine in goats undergoing elective stifle arthrotomy. The Veterinary journal. 2011 Apr;188(1):53-7.

8. Biboulet $\mathrm{P}$, Morau D, Aubas P, Bringuier-Branchereau S, Capdevila X. Postoperative analgesia after total-hip arthroplasty: comparison of intravenous patient-controlled analgesia with morphine and single injection of femoral nerve or psoas compartment block. a prospective, randomized, double-blind study. Regional Anesthesia and Pain Medicine. 2004 Apr;29(2):102-109.

9. Marino J, Russo J, Kenny M, Herenstein R, Livote E, Chelly J. Continuous lumbar plexus block for postoperative pain control after total hip arthroplasty. A randomized controlled trial. The Journal of bone and joint surgery American volume. 2009 Jan;91(1):29-37.

10. Capdevila $X$. Surgical Outcome and Duration $\mathrm{o}$ after $\mathrm{M}$ a $\mathrm{j} \circ \mathrm{r}$ Knee Surgery. Anesthesiology. 1999;91(1):8-15. 
11. Singelyn FJ, Deyaert M, Joris D, Pendeville E, Gouverneur JM. Effects of intravenous patient-controlled analgesia with morphine, continuous epidural analgesia, and continuous three-in-one block on postoperative pain and knee rehabilitation after unilateral total knee arthroplasty. Anesthesia \& Analgesia. 1998;87:88-92.

12. Siddiqui ZI, Cepeda MS, Denman W, Schumann R, Carr DB. Continuous lumbar plexus block provides improved analgesia with fewer side effects compared with systemic opioids after hip arthroplasty: a randomized controlled trial. Regional anesthesia and pain medicine. 2007;32(5):393-8.

13. Naja ZM, Raf M, El Rajab M, et al. Nerve stimulator-guided paravertebral blockade combined with sevoflurane sedation versus general anesthesia with systemic analgesia for postherniorrhaphy pain relief in children: a prospective randomized trial. Anesthesiology. 2005 Sep;103(3):600-5.

14. Macfarlane AJR, Prasad GA, Chan VWS, Brull R. Does regional anaesthesia improve outcome after total hip arthroplasty? A systematic review. British journal of anaesthesia. 2009 Sep;103(3):335-45.

15. Ilfeld BM, Mariano ER, Girard PJ, et al. A multicenter, randomized, triple-masked, placebo-controlled trial of the effect of ambulatory continuous femoral nerve blocks on discharge-readiness following total knee arthroplasty in patients on general orthopaedic wards. Pain. 2010 Sep;150(3):477-84.

16. Jones RS. Epidural analgesia in the dog and cat. Veterinary journal (London, England: 1997). 2001 Mar;161(2):123-31.

17. Mahler SP, Adogwa AO. Anatomical and experimental studies of brachial plexus, sciatic, and femoral nerve-location using peripheral nerve stimulation in the dog. Veterinary anaesthesia and analgesia. 2008 Jan;35(1):80-9.

18. Portela D, Melanie P, Briganti a, Breghi G. Nerve stimulator-guided paravertebral lumbar plexus anaesthesia in dogs. Veterinary research communications. 2008 Sep;32 Suppl 1:S307-10.

19. Aithal HP, Pratap K, Singh GR. Clinical effects of epidurally administered ketamine and xylazine in goats. Small Ruminant Research. 1996;24:55-64.

20. Mpanduji DG, Mgasa MN, Bittegeko SB, Batamuzi EK. Comparison of xylazine and lidocaine effects for analgesia and cardiopulmonary functions following lumbosacral epidural injection in goats. Zentralblatt für Veterinärmedizin Reihe A. 1999 Dec;46(10):605-11.

21. Vesal N, Oloumi MM. A preliminary comparison of epidural lidocaine and xylazine during total intravenous anaesthesia in Iranian fat-tailed sheep. Zentralblatt für Veterinärmedizin Reihe A. 1998 Sep;45(6-7):353-60. 
22. Wagner $\mathrm{AE}$, Dunlop $\mathrm{Cl}$, Turner a $\mathrm{S}$. Experiences with morphine injected into the subarachnoid space in sheep. Veterinary surgery: VS. 1996;25(3):256-60.

23. DeRossi R, Pagliosa R, Módolo TC, Maciel FB, Macedo GG. Thoracic epidural analgesia via the lumbosacral approach using multiport catheters with a low concentration of bupivacaine and morphine in sheep. Veterinary anaesthesia and analgesia. 2012 Mar;39(3):306-14.

24. Krohm $\mathrm{P}$, Levionnois $\mathrm{O}$, Ganster M, Zilberstein L, Spadavecchia C. Antinociceptive activity of pre- versus post-operative intra-articular bupivacaine in goats undergoing stifle arthrotomy. Veterinary anaesthesia and analgesia. $2011 \mathrm{Jul} ; 38(4): 363-73$.

25. Shafford HL, Hellyer PW, Turner AS. Intra-articular lidocaine plus bupivacaine in sheep undergoing stifle arthrotomy. Veterinary anaesthesia and analgesia. 2004 Jan;31(1):206.

26. Bailie DS, Ellenbecker TS. Severe chondrolysis after shoulder arthroscopy: a case series. Journal of shoulder and elbow surgery / American Shoulder and Elbow Surgeons . [et al]. 2009;18(5):742-7.

27. Abdallah FW, Brull R. Is sciatic nerve block advantageous when combined with femoral nerve block for postoperative analgesia following total knee arthroplasty? A systematic review. Regional anesthesia and pain medicine [Internet]. 2011 Jan [cited 2013 Mar 5];36(5):493-8. Available from: http://www.ncbi.nlm.nih.gov/pubmed/21857266

28. Campoy L, Martin-Flores M, Ludders JW, Erb HN, Gleed RD. Comparison of bupivacaine femoral and sciatic nerve block versus bupivacaine and morphine epidural for stifle surgery in dogs. Veterinary anaesthesia and analgesia [Internet]. 2012 Jan [cited 2013 May 11];39(1):91-8. Available from: http://www.ncbi.nlm.nih.gov/pubmed/22117792

29. Kinzel S, Fasselt R, Prescher A, Selzer C, Graf von Keyserlingk D, Küpper W. [Sensory innervation of the hip joint capsule in dogs]. Tierärztliche Praxis Ausgabe $K$, Kleintiere/Heimtiere. 1998 Sep;26(5):330-5.

30. Birnbaum K, Prescher a, Hessler S, Heller KD. The sensory innervation of the hip joint-an anatomical study. Surgical and radiologic anatomy: SRA. 1997 Jan;19(6):371-5.

31. Rankin JS, Diesem CD. Innervation of the equine hip and stifle joint capsules. Journal of the American Veterinary Medical Association. 1976 Sep;169(6):614-9.

32. Ben-Ari AY, Joshi R, Uskova A, Chelly JE. Ultrasound localization of the sacral plexus using a parasacral approach. Anesthesia and analgesia. 2009 Jun;108(6):1977-80. 
33. Portela DA, Otero PE, Tarragona L, Briganti A, Breghi G, Melanie P. Combined paravertebral plexus block and parasacral sciatic block in healthy dogs. Veterinary anaesthesia and analgesia. 2010 Nov;37(6):531-41.

34. Ho AMH, Karmakar MK. Combined paravertebral lumbar plexus and parasacral sciatic nerve block for reduction of hip fracture in a patient with severe aortic stenosis. Canadian journal of anaesthesia = Journal canadien d'anesthésie. 2002 Nov;49(9):94650 .

35. Troncy E, Junot S, Keroack S, et al. Results of preemptive epidural administration of morphine with or without bupivacaine in dogs and cats undergoing surgery: 265 cases (1997-1999). Journal of the American Veterinary Medical Association. 2002 Sep;221(5):666-72.

36. Portela DA, Otero PE, Tarragona L, et al. Combined paravertebral plexus block and parasacral sciatic block in healthy dogs. Veterinary anaesthesia and analgesia. 2010 Nov;37(6):531-41.

37. Schroeder CA, Snyder LBC, Tearney CC, Baker-Herman TL, Schroeder KM. Ultrasound-guided transversus abdominis plane block in the dog: an anatomical evaluation. Veterinary anaesthesia and analgesia. 2011 May;38(3):267-71.

38. Benigni L, Corr SA, Lamb CR. Ultrasonographic Assessment of the Canine Sciatic Nerve. Veterinary Radiology \& Ultrasound. 2007 Sep;48(5):428-433.

39. Shilo $Y$, Pascoe PJ, Cissell D, et al. Ultrasound-guided nerve blocks of the pelvic limb in dogs. Veterinary anaesthesia and analgesia. 2010 Sep;37(5):460-70.

40. Costa-Farré C, Blanch XS, Cruz JI, Franch J. Ultrasound guidance for the performance of sciatic and saphenous nerve blocks in dogs. Veterinary journal (London, England: 1997). 2011 Feb;187(2):221-4.

41. Echeverry DF, Gil F, Laredo F, et al. Ultrasound-guided block of the sciatic and femoral nerves in dogs: a descriptive study. Veterinary journal (London, England: 1997). 2010 Nov;186(2):210-5.

42. Marhofer $\mathrm{P}$, Greher $\mathrm{M}$, Kapral $\mathrm{S}$. Ultrasound guidance in regional anaesthesia. $\mathrm{Br} \mathrm{J}$ Anaesth 2005;94(1):7-17.

43. Campoy L, Martin-Flores M, Looney AL, et al. Distribution of a lidocaine-methylene blue solution staining in brachial plexus, lumbar plexus and sciatic nerve blocks in the dog. Veterinary anaesthesia and analgesia. 2008 Jul;35(4):348-54.

44. Popesko P. Atlas der topographischen Anatomie der Haustiere: Band 3. Becken und Gliedmaßen. Enke; 1993. 
45. König, Liebich. Anatomie der Haussäugetiere: Lehrbuch und Farbatlas für Studium und Praxis + Anatomie online: die Bilddatenbank mit dem Plus. Schattauer Verlag; 2011.

46. Böhme G. Lehrbuch der Anatomie der Haustiere: Nervensystem, Sinnesorgane, endokrine Drüsen. Georg Thieme Verlag; 2003.

47. Ben-Ari AY, Joshi R, Uskova A, Chelly JE. Ultrasound localization of the sacral plexus using a parasacral approach. Anesthesia and analgesia. 2009 Jun;108(6):1977-80.

48. Shilo Y, Pascoe PJ, Cissell D, Johnson EG, Kass PH, Wisner ER. Ultrasound-guided nerve blocks of the pelvic limb in dogs. Veterinary anaesthesia and analgesia. 2010 Sep;37(5):460-70.

49. Rasmussen LM, Lipowitz AJ, Graham LF. Development and verification of saphenous, tibial and common peroneal nerve block techniques for analgesia below the thigh in the nonchondrodystrophoid dog. Veterinary anaesthesia and analgesia. 2006 Jan;33(1):3648.

50. Campoy L, Bezuidenhout AJ, Gleed RD, Martin-Flores M, Raw RM, Santare CL, et al. Ultrasound-guided approach for axillary brachial plexus, femoral nerve, and sciatic nerve blocks in dogs. Veterinary anaesthesia and analgesia. 2010 Mar;37(2):144-53.

51. Campoy L, Bezuidenhout AJ, Gleed RD, et al. Ultrasound-guided approach for axillary brachial plexus, femoral nerve, and sciatic nerve blocks in dogs. Veterinary anaesthesia and analgesia. 2010 Mar;37(2):144-53.

52. Wagner AE et al. Evaluation of effects of sciatic and femoral nerve blocks in sheep undergoing stifle surgery. Labanimal. 2011;11(5).

53. McNamee $\mathrm{D}$ a, Parks L, Milligan KR. Post-operative analgesia following total knee replacement: an evaluation of the addition of an obturator nerve block to combined femoral and sciatic nerve block. Acta anaesthesiologica Scandinavica. 2002 Jan;46(1):95-9.

54. Böhme G. Lehrbuch der Anatomie der Haustiere: Nervensystem, Sinnesorgane, endokrine Drüsen. Georg Thieme Verlag; 2003.

55. Luyet $\mathrm{C}$, Eichenberger $\mathrm{U}, \mathrm{Greif} \mathrm{R}$, et al. Ultrasound-guided paravertebral puncture and placement of catheters in human cadavers: an imaging study. British journal of anaesthesia. 2009 Jan;102(4):534-9.

56. Sites B, Gallagher J, Cravero J, Lundberg J, Blike G. The learning curve associated with a simulated ultrasound-guided interventional task by inexperienced anesthesia residents. Regional Anesthesia and Pain Medicine. 2004 Dec;29(6):544-548. 
455

456

457

458

459

460

461

462

463
57. Sala-Blanch XMD et al. Intraneural Injection during Anterior Approach for Sciatic Nerve Block. Anesthesiology. 2004;101:1019-1030.

58. Sala-Blanch X, Pomés J, Matute $\mathrm{P}$, et al. Intraneural Injection during Anterior Approach for Sciatic Nerve Block. Anesthesiology. 2004;101:1019-1030.

59. Davies a F, Segar EP, Murdoch J, Wright DE, Wilson IH. Epidural infusion or combined femoral and sciatic nerve blocks as perioperative analgesia for knee arthroplasty. British journal of anaesthesia. 2004 Sep;93(3):368-74. 


\section{$464 \quad$ Figures}

465 Fig. 1: Ultrasonographic image of the left sciatic nerve (a) near the greater sciatic foramen.

$466 \quad$ Needle (b) with its reflector mark and the ilium (c).

467

468 Fig. 2: Ultrasonographic image of the right femoral nerve, the femoral artery and the femoral 469 vein (d). The needle tip can be seen underneath the fascia of the sartorius muscle near the 470 femoral nerve, the femoral artery and the femoral vein. The sartorius muscle with its triangular 471 appearance (a), the femur (b) and the needle (c).

473 Fig. 3: Left pelvis, dorsal view. The sheep cadaver was positioned in sternal recumbency. The left sciatic nerve (a) and the surrounding tissue have been stained with methylene blue.

Fig. 4: Right pelvic limb, medial view. The cadaver was positioned in supine position. The left femoral nerve and the surrounding tissue have been stained with methylene blue. 


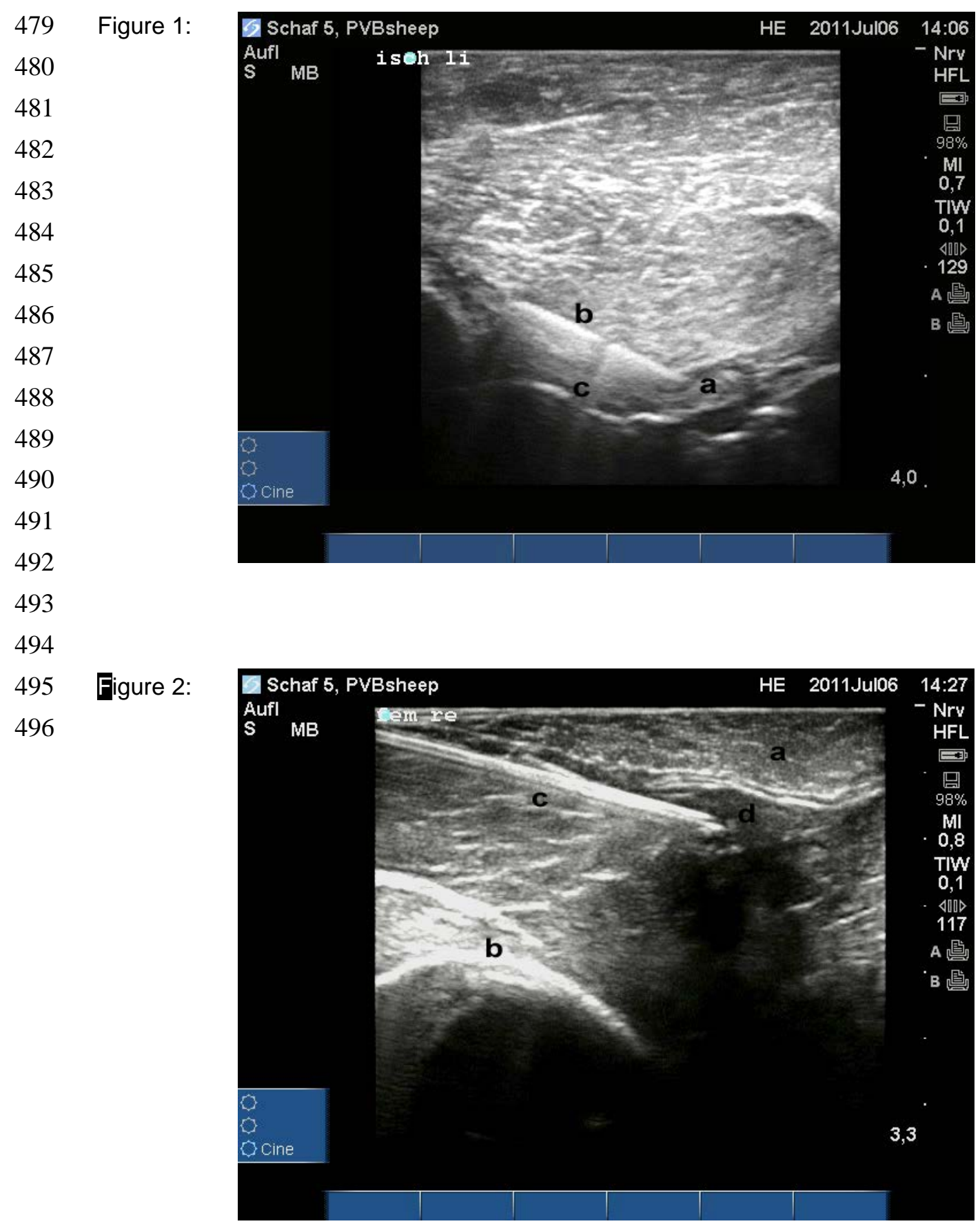




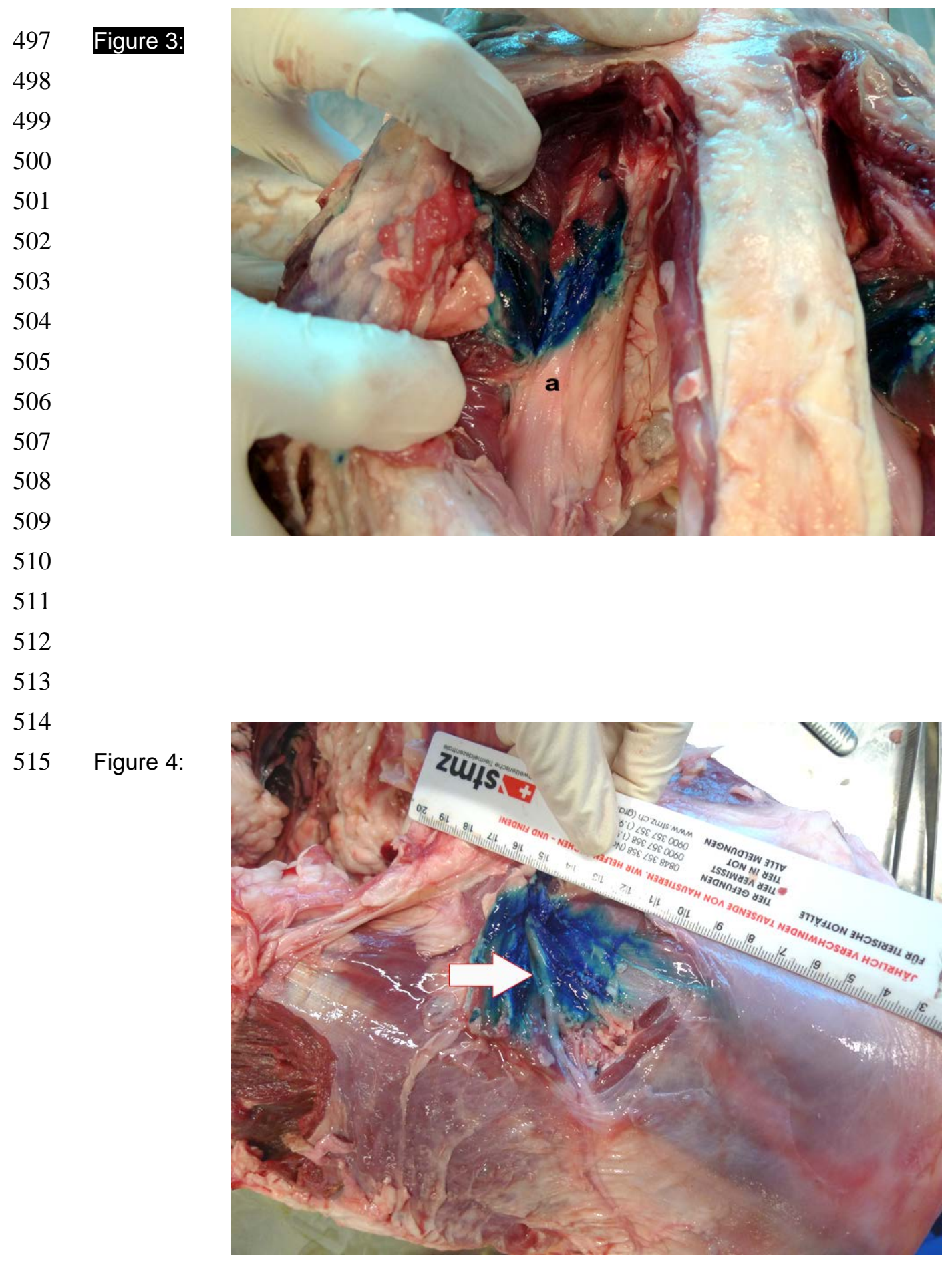

